\title{
A volume-weighted-average-price (VWAP) method for estimating beta in the context of reference-day risk
}

\author{
Keshav Sahadev \\ Mike Ward \\ Chris Muller
}

Gordon Institute of Business Science

University of Pretoria

South Africa

Partially funded by a National Research Foundation Grant

February 2018

\begin{abstract}
The ability to accurately estimate systematic risk (or beta) when reference-day risk is considered is an ineluctable requirement for all applications of the capital asset pricing model (CAPM).

This research documents evidence of reference-day risk for shares on the Johannesburg All Share Index. In response to the need for greater accuracy when estimating systematic risk, this paper contributes a volumeweighted-average-price (VWAP) method for estimating beta which may be employed when reference-day risk is considered.

Furthermore, this research applies a graphical time-series approach to test the underlying risk-reward tenet postulated by the CAPM. Using beta as a measure of systematic risk, this research finds that the CAPM appears to imperfectly specify the risk-reward trade-off.
\end{abstract}

Keywords: Beta, reference-day risk, VWAP 


\section{Introduction}

The risk-reward tradeoff has been a prominent focus for empirical finance literature and investment practitioners since the 1950s. The parsimony of this risk-reward relationship is intuitively captured by the capital asset pricing model (CAPM), jointly ascribed to Markowitz (1952; 1956; 1959), Treynor (1961), Sharpe (1964), Lintner (1965) and Mossin (1966) for their respective contributions to its development.

The CAPM describes a simple linear model for estimating the expected return on an asset in terms of its systematic risk, and by doing so, provides a formal relationship between risk and return (Fama \& French, 2004; Ward \& Muller, 2012). The CAPM can be expressed as:

$$
E\left(R_{i}\right)=R_{f}+\beta_{i}\left(E\left(R_{m}\right)-R_{f}\right)
$$

(Equation 1)

Where;

$\mathbf{E}\left(\mathbf{R}_{\mathbf{i}}\right)$ is the expected return on the share (asset),

$\mathbf{R}_{\mathbf{f}}$ is the risk-free rate of interest, such as interest arising from government bonds,

$\mathbf{E}\left(\mathbf{R}_{\mathbf{m}}\right)$ is the expected return of the market, and

$\boldsymbol{\beta}_{\mathrm{i}}$ is the "beta" (a term originally coined by Sharpe (1964)) and represents the systematic risk component of an asset, as specified by the CAPM. In essence, beta describes the sensitivity of the expected excess returns of an asset to the expected excess returns earned by the market, and is expressed as;

$$
\beta_{\mathrm{i}}=\frac{\operatorname{Cov}\left(\mathrm{R}_{\mathrm{i}}, \mathrm{R}_{\mathrm{m}}\right)}{\operatorname{Var}\left(\mathrm{R}_{\mathrm{m}}\right)}
$$

(Equation 2)

where $\operatorname{Cov}\left(\mathrm{R}_{\mathrm{i}}, \mathrm{Rm}_{\mathrm{m}}\right)$ is the covariance between the return on asset $i$ and the return on the market portfolio, using historical data (Chen \& Reeves, 2012; Heymans \& Brewer, 2015). Simply stated, the CAPM emphasises that the risk of an asset is a function of its beta (Gonzalez, Rodriguez \& Stein, 2014).

Equivalently, Fama and Macbeth (1973) suggest a method for estimating beta by using a rolling linear regression of individual share returns on market returns (monthly), typically over a five-year period. By convention, monthly returns are estimated by constructing a series of consecutive monthly closing prices, typically using the last date of each month as data points in the series, over a five-year period. Thereafter, each of the monthly share and market returns are computed by estimating the log returns between the closing prices over the period. This method is commonly referred to as the ordinary least squares (OLS) regression method. For the purposes of this paper, we will refer to the OLS regression method as the convention for estimating beta.

Since its development, the CAPM in its simplicity and intuition, was not earnestly questioned until the publication of a paper by Fama and French (1992), who suggested that if assets are priced rationally, then share risks are not one-dimensional (i.e. exclusively a function of beta) but rather, multi-dimensional. This finding was radical in the field of systematic risk estimation, especially given the extensive use of beta and the CAPM in academia and practice. Despite the theoretical and practical appeal of the CAPM however, the empirical evidence in support of the model is at best, weak (Ward \& Muller, 2012). Most of this criticism has emanated from investigations focused on beta. Studies conducted by Fabozzi and Francis (1978), Collins, Ledolter and Rayburn (1987), Faff and Brooks (1996), and Brooks, Faff, Gangemi and Lee (1997) have analysed a variety of financial markets and found evidence of beta instability. In other words, the findings suggest that beta coefficients move randomly through time rather than remain stable, as the OLS regression model presumes. Furthermore, Novak (2015) suggests that the conventional method for estimating beta has also been criticised for low explanatory power in explaining share returns. Along with the estimation criticism, Fama and French (1992; 2004), van 
Rensburg and Robertson (2003), Montier (2009), Strugnell, Gilbert and Kruger (2011) and Ward and Muller (2012), suggest that there is essentially little to no evidence of a positive, linear relationship between systematic risk and returns. In contrast, research has found that there is an inverse relationship between systematic risk and return when one approximates systematic risk using beta, a finding which is unequivocally contradictory to the underlying tenet of the CAPM (Montier, 2009; Ward and Muller, 2012). According to Novak (2015), the wave of literature documenting the low explanatory power of beta and the inverse relationship documented by some authors has led some to proclaim beta "dead". One of the foremost criticisms against beta is the concept of reference-day risk.

In a seminal paper, Acker and Duck (2007) introduced the concept of "reference-day risk" to refer to large sampling variations and estimation risks that can be detected in share returns, variances and share betas, and which is simply attributable to the choice of an initial reference day when calculating monthly share returns. In summary, reference-day risk creates additional uncertainty for investors and finance practitioners and leads to inadvertent or unwarranted outcomes based on beta estimates. As a result, reference-day risk has become one of the most preeminent research areas to gain momentum in recent years, as it has significant implications for accurate beta estimation. Investigations conducted by Acker and Duck (2007), Dimitrov and Govindaraj (2007), Gonzalez et al (2014), and Baker, Rajaratnam and Flint (2016) have all evidenced that by using any five-year sample, and selecting one day of the first month as the reference day to construct a series of monthly returns, different choices for the reference day produce large variations in estimated betas.

The findings from the original investigation by Acker and Duck (2007) have prompted significant research into developing and testing alternative, independent-reference-day methods for estimating beta. Among the beta estimation methods tested, were the Blume (1971) regression method, the Dimson (1979) adjustment for thin trading, the Vasicek (1973) Bayesian beta estimate and the $t$-distribution method for adjusting beta as developed by Cademartori, Romo, Campos and Galea (2003). Out of these, only the method prescribed by Cademartori et al (2003) yielded any promising result for accurately estimating systematic risk when reference-day risk is considered.

This paper aims to resurrect the systematic risk parameter, beta, by contributing a volume-weighted-averageprice (VWAP) estimate for beta which can be employed in the context of reference day risk. To achieve this, this research first evidences the degree to which reference-day risk leads to variations in beta for shares on the JSE ALSI. Thereafter, estimates of beta using the VWAP methodology are statistically tested to determine the robustness and applicability of applying the VWAP method as a variation to the conventional method of estimating beta.

Lastly, this research transcends prior research investigations of a similar nature by determining whether the reference-day-independent beta adheres to the underlying tenets of the CAPM. This is achieved by employing a graphical time-series analysis to observe whether VWAP betas are a more reliable representation of systematic risk in the risk-return tradeoff.

Consequently, an appropriate methodology is derived to test each of these objectives.

This paper is therefore organised as follows. The following section reviews the literature on the subject. Thereafter, the sample, methodology and results are detailed and discussed. The last section offers concluding comments and guidelines for future research.

\section{Literature review}

Reference-day risk and the mitigation of it thereof, is one of the most contemporary issues contributing to financial literature intended for accurate beta measurement. The results of the original investigation by Acker and Duck (2007) have prompted significant research into developing and testing alternative, independentreference-day methods for estimating beta. Among the methods most tested were the Blume (1971) regression 
method, the Vasicek (1973) Bayesian beta and the Dimson (1979) adjustment for thin trading. This section presents some of the empirical literature describing reference-day risk and the variations that different choices of reference day have on beta estimates. We begin with a brief overview of the three commonly used adjustment methods.

\section{The Blume regression method for estimating beta}

In 1971, Marshall E. Blume set out to examine the stationarity of the conventional beta estimate over time and to propose a method of obtaining improved assessments of systematic risk. By using the conventional estimation method, Blume (1971) created portfolios of shares according to the magnitude of estimated betas. Blume then estimated the betas for the resultant portfolios across two, non-overlapping consecutive periods. Blume's results indicate a tendency for the high-risk portfolios (i.e. portfolios with the high beta estimates) to decline monotonically towards a mean of 1 , whereas the lower-risk portfolios tend upwards towards a mean of 1 over time (Blume, 1971; Baker et al, 2016).

Blume (1971) uses the betas generated from the two consecutive, non-overlapping periods to estimate "predicted betas" for a third, non-overlapping period. This is done by regressing the estimated values of beta $\left(\beta_{i}\right)$ in one period on the values estimated in the previous period $\left(\beta_{i-1}\right)$ to yield a modified estimate for the assessment of future systematic risk. This relationship is represented by the equation;

$$
\beta_{\mathrm{i}}=a+b \beta_{\mathrm{i}-1}+e_{\mathrm{t}} \quad \text { (Equation 3) }
$$

where;

$\beta_{i}$ is the beta in one period,

$\beta_{i-1}$ is the beta in the preceding period,

$a$ and $b$ are regression coefficients, and

$e_{t}$ is a zero-mean error term (Baker et al, 2016).

The mean squared errors for the adjusted and unadjusted beta estimates are compared to the beta estimates generated using the conventional estimation method. The research conducted by Blume (1971) concludes that for both individual shares and portfolios of two or more shares, the estimates adjusted for the historical rate of regression are more accurate than the unadjusted estimates.

\section{The Dimson adjustment for thin trading}

Dimson (1979) uses the findings of Fisher (1966) to propose an adjustment method to beta estimation when shares are subject to infrequent trading. Fisher (1966) suggests that a major source of bias in estimating beta emanates from the tendency of shares which are subject to thin trading having substantially underestimated covariances. Fisher (1966) also proved that the downward bias in the covariance of frequently traded shares is less significant when compared to infrequently traded shares. The findings of this investigation formed the premise for Dimson's (1979) Aggregate Coefficients (AC) method for estimating the systematic risk of a share.

Dimson's (1979) AC method asserts that the true systematic risk parameter beta $(\hat{\beta})$ can be obtained from price data which is subject to infrequent trading by regressing observed share returns on lagged, synchronous and leading market returns. The regression equation is given by the expression;

$$
\widehat{R}_{t}=\hat{\alpha}+\sum_{k=-n}^{n} \widehat{\beta}_{k} \widehat{M}_{t+k}+\varepsilon_{t},
$$


where;

$\widehat{R}_{t}$ represents the observed share returns,

$M_{\mathrm{t}+\mathrm{k}}$ represents the lagged, synchronous and leading market returns

$\hat{\alpha}$ and $\widehat{\beta}_{k}$ are the estimated intercept and slope coefficients, respectively and

$\varepsilon_{\mathrm{t}}$ is a zero-mean error term (Dimson, 1979).

Subsequently, the systematic risk parameter beta $(\beta)$ is calculated as the sum of the aggregate coefficients in Equation 5. Thus,

$$
\hat{\beta}=\sum_{k=-n}^{n} \widehat{\beta}_{k}
$$

(Equation 6)

Dimson (1973) compares the beta estimates derived using the AC method against the conventional regression method. After controlling for and simplifying assumptions to allow comparison across methods, Dimson concludes that the AC method is a more efficient beta estimation method as the conventional regression method is significantly biased for shares which are thinly traded.

\section{The Vasicek Bayesian method for beta estimation}

Using Bayesian Decision Theory, Vasicek (1973) presented a method for generating Bayesian estimates for beta. According to Vasicek (1973), Bayesian Decision Theory provides formal procedures which makes use of information available prior to sampling, in conjunction with the sample information, to construct optimal estimates which minimise the expected error.

In deriving the Bayesian beta estimate, Vasicek (1973) argues that the properties of the standard OLS beta parameter do not reasonably reflect the required properties of a beta estimator. Specifically, the conventional estimator assumes the property;

$$
E(b \mid \beta)=\beta
$$

which describes the mean value of beta, with the conjoint assumption that the true value for beta is known. Vasicek (1973) challenges the assumption that the true value of beta is known and described by the mean of the estimator. In other words, one would not require an estimator $(b)$ if the true value of beta $(\beta)$ is known. Vasicek (1973) therefore hypothesises that the reverse of this is true; it is the sample coefficient (b) which is known and on this basis, one can infer about the distribution of the parameter, beta $(\beta)$.

Vasicek (1973) therefore suggests that given the normal prior distribution with mean $b^{\prime}$ and variance $s^{\prime}{ }_{b}$, the distribution of $\beta$ is approximately normal, with mean $b "$ and variance $s^{\prime \prime}{ }_{b}$, and $b$ is the conventional estimate of systematic risk and;

$$
\begin{array}{cc}
b^{\prime \prime}=\frac{\left(b^{\prime} / s^{\prime}{ }_{\mathrm{b}}+b / s^{2}\right)}{\left(1 / s^{\prime}{ }_{\mathrm{b}}+1 / s^{2} \mathrm{~b}\right)}, & s^{2} \mathrm{~b}=\frac{s^{2}}{\sum\left(M_{\mathrm{t}}-\bar{M}\right)^{2}}, \\
s^{\prime \prime 2}{ }_{\mathrm{b}}=\frac{1}{\left(1 / s^{\prime 2} \mathrm{~b}+1 / s^{\mathrm{b}} \mathrm{b}\right.}, & s^{2}=\frac{\sum\left(R_{\mathrm{t}}-\widehat{\alpha}-b M_{\mathrm{t}}\right)^{2}}{(T-2)},
\end{array}
$$

where $t$ is the time step, with $t=1,2, \ldots, T$ (Baker et al, 2016). 
According to Vasicek (1973), the Bayesian estimate for the systematic risk parameter, beta, is given by the mean of the distribution $b$ " and describes the knowledge about the distribution of the estimated beta, given the information from the sample and the prior information.

Lastly, Vasicek (1973) asserts that Bayesian estimates are preferred to the conventional beta estimates as the Bayesian procedure minimises the loss of accuracy arising from misestimation. In contrast, the conventional estimates minimise the error of sampling. Furthermore, in addition to the sample information, the Bayesian estimates incorporates prior information is the estimation of systematic risk (Vasicek, 1973).

\section{Estimating beta when reference-day risk is considered}

Acker and Duck (2007) were the first to demonstrate the existence of reference-day risk associated with monthly returns implicit in shares listed on the S\&P500 Index and set out to explore the level of reference-day risk implicit in estimates of beta.

Using data from a sample of 459 companies sourced through Datastream, Acker and Duck (2007) examined data for a 15 year period, across three five-year sub-intervals. They prove that estimates of beta are highly sensitive to the choice of reference day. In extreme cases, the choice of reference-day can significantly amplify the estimate of a share's beta as well as alter the sign of beta estimates. Drawing on the sample of listed companies on the S\&P500, the authors present evidence of a share's beta falling by 0.931 and rising by 3.454 depending on the choice of reference-day. Perhaps more pertinent to this paper, is the finding that approximately $75 \%$ of all observed betas estimated using the conventional methodology could be classified as either positive or negative by an appropriate choice of reference day. This finding has profound ramifications for both academia and industry, as it renders risk-adjusted asset selection using conventional beta estimates impractical, inconsequential and insufficient for portfolio construction.

Acker and Duck (2007) thereafter commence with investigating whether the Blume (1971) regression method, the Vasicek (1973) Bayesian beta and the Dimson (1979) adjustment for thin trading reduces the variation and range of beta estimates for which the unadjusted beta is highly sensitive to the choice of reference day.

The authors report that the Blume (1971) regression method reduces only the most severe reference-day variability. Similarly, the Vasicek (1973) Bayesian estimation method also reduces the range and variation of beta estimates for shares which have high sensitivity to the selection of a reference day (Acker \& Duck, 2007). However, both estimation methods exhibit sensitivity to the choice of reference day (although this variation is less marked for estimates of Blume betas). The findings imply that estimated betas using both the Blume (1971) and Vasicek (1973) adjustments are indeed subject to reference-day risk, causing shares to be incorrectly classified as either aggressive or defensive based on the selection of an initial reference day (Acker \& Duck, 2007).

Lastly, even though the investigation evidenced the presence of reference-day risk for both individual shares and equity indices, Acker and Duck (2007) hypothesise that the effect of reference-day risk may be amplified by thin trading. Accordingly, the authors apply the Dimson (1979) adjustment method for thin trading in estimating betas in the context of reference-day risk. The results of the investigation suggest that the Dimson (1979) adjustment method only slightly reduces the variability of beta estimates for the highest ranges. The authors also observe a tendency for estimated betas to exhibit significant variation based on the choice of reference day, indicating that the Dimson (1979) adjustment does not yield any significant difference in estimating systematic risk when reference-day risk is observed.

Dimitrov and Govindaraj (2007) set out to investigate the findings from Acker and Duck's (2007) study from a data dependency perspective. They argue that Acker and Duck's (2007) sample (which was drawn from Datastream) may not be applicable for the United States context and propose replicating the analysis using data sourced from the Centre for Research in Security Prices (CRSP). Furthermore, Dimitrov and Govindaraj (2007) 
expand the study by Acker and Duck (2007) by investigating the existence of reference-day risk using daily returns (as opposed to monthly returns) over a five-year period.

Using data sourced from the CRSP from the period January 1995 to December 1999, Dimitrov and Govindaraj (2007) use daily dividend-adjusted share returns to construct a series of 60 monthly returns across 19 different reference days, for each of 439 sample companies. The investigation verifies the existence of reference-day risk in companies listed on the S\&P500, using data sourced from the CRSP (Dimitrov \& Govindaraj, 2007). Dimitrov and Govindaraj (2007) also report that betas estimated from the sample exhibit significant variation when different choices of the initial reference day are used in the computation. Lastly, the findings from the investigation suggest that reference day risk is implicit for both individual shares and market indices.

Gonzalez et al (2014) explicate the research conducted by both Acker and Duck (2007) and Dimitrov and Govindaraj (2007) by expanding the data dependency and methodology parameters employed in the previous studies. Gonzalez et al (2014) compare the $t$-distribution method for adjusting beta as developed by Cademartori et al (2003) to the conventional method and Blume's (1971) regression method for estimating beta.

According to Gonzalez et al (2014), the $t$-distribution method proposes a replacement of the standard normal distribution with the Student's $t$, which is a symmetric distribution with heavier tails than the normal distribution. Gonzalez et al (2014) purport that the heavier tails in the Student's $t$ are more appropriate for estimating beta when reference-day risk is considered, as it more appropriately compensates for the error term in the linear regression used to estimate betas. Furthermore, Cademartori et al (2003) prove that the $t$-distribution method for adjusting beta is better able to incorporate the influence of outliers in estimating beta. Lastly, Blume's (1971) method was selected by Gonzalez et al (2014) for comparison to the $t$-distribution method, as it was the method which reduced the effect of reference-day risk the most in Acker and Duck's (2007) investigation.

Gonzalez et al (2014) select a sample of 1563 shares, traded on the NYSE, AMEX and NASDAQ exchanges obtained from the CRSP, for the period 2007 to 2011 and report that the choice of reference day results in significant variations in estimated betas. Gonzalez et al (2014) also report that betas estimated using Blume's (1971) regression method exhibited significant variations across different reference days. Using the $t$-distribution method however, they record that the choice of reference day becomes less significant citing that the larger recorded ranges decreased significantly for the betas. They conclude that the $t$-distribution method for adjusting beta most significantly reduces reference-day variation when compared to Blume's (1971) method and the conventional method.

More recently, Baker et al (2016) set out to establish the existence of reference-day risk in the JSE Top 40. Baker et al (2016) use daily closing levels of the ALSI and closing prices for shares making up the JSE Top 40 index for the period January 2000 to July 2015, sourced from Datastream.

As observed by Acker and Duck (2007), Dimitrov and Govindaraj (2007) and Gonzalez et al (2014), the authors indicate that reference-day risk exists on the JSE op 40 and creates additional uncertainty for investors intending to create share portfolios, valuing companies or managing capital (Baker et al, 2016). The authors also test the Blume (1971), Dimson (1979) and Vasicek (1973) Bayesian methods for adjusting beta to investigate whether the adjusted betas exhibit lower reference-day ranges than the conventional betas (Baker et al, 2016).

In estimating the betas calculated using Blume's (1971) regression method, Baker et al (2016) report that the Blume-adjusted betas increase the variation and range of beta estimates for 19 out of 31 companies and conclude that the Blume (1971) regression method does not consistently and considerably reduce the variation and range for betas when reference day risk is observed. Similarly, the average range for the Dimson-adjusted betas was significantly larger than that of the conventional betas. The range for beta estimates using the Dimson (1979) adjustment increased for 30 out of the 40 companies sampled, leading the authors to conclude that the Dimson (1979) adjustment for thin trading actually pronounces reference day variation in systematic risk 
estimates. Lastly, the authors report that the Vasicek (1973) Bayesian adjustment method was the only estimation method yielding any promising results, with 35 out of the JSE Top 40 companies exhibiting lower reference-day ranges than the conventional betas.

Baker et al (2016) then introduce the prospect of using a nonparametric bootstrap method to determine beta estimates which are independent of the reference-day. Given that there are at least 20 trading days in every month, the authors first organise share price and index level data in series, according to each of the 20 trading days, with the first trading day not necessarily corresponding to the first calendar day of the month. Using each series, Baker et al (2016) estimate betas for each share using the conventional methodology (i.e. using 60 months data) across the 20 different trading days to bootstrap a beta distribution for the companies under investigation. In other words, assuming that a share has a value for beta which is not directly observable in the market, the authors simulate returns data for each company and the market index using the correlation relationship for the observed beta distribution. Baker et al (2016) simulate 60 returns-paired sequences (for the company and the market index) representing 60 months. This process assumes an arbitrary reference day, allowing for the estimation of share betas for each of the 60 returns sequences. The authors then select 20 such sequences for which the variance of beta is maximised (giving the largest possible range). Lastly, they then select a random share price and use the random sequence of share and index returns to calculate "bootstrapped betas".

Using this method, the authors note that for shares on the JSE Top 40, the expected value of a reference-day independent beta (i.e. the bootstrapped beta) was approximately equal to the average of the 20 betas estimated for each day using the conventional method. Baker et al (2016) propose that the mean value of the bootstrapped beta distribution therefore provides a reference-day independent estimate of systematic risk for a particular share. Even though the results of the investigation do not yield the desired result, the authors do note some benefits of employing this method in estimating beta (See Baker et al, 2016).

In summary, the literature discussed above has not revealed a methodology which is independent of the reference-day problem in beta estimation. The final component of this theoretical discussion now focuses on the relevant literature regarding the proposed VWAP methodology.

\section{Empirical evidence of VWAP as a method to eliminate the reference-day problem}

The is little to no literature available on estimating beta through employing a VWAP adjustment or VWAP methodology in the context of reference-day risk. However, perhaps the most pertinent literature is presented by Ting $(2005 ; 2006)$ who suggests that a VWAP is closer to the equilibrium price of a share than the daily closing price. Ting $(2005 ; 2006)$ bases this on the premise that VWAP considers all the intra-day prices at which transactions have occurred and evidences that daily returns computed with VWAP have a smaller realised variance than that with the closing price. Moreover, Ting (2006) concludes that the variance spread between VWAP and the closing price is economically significant and has implications for performance measurement and pricing of derivatives.

More critical to this research however, Ting (2006) suggests that relative to the volatility of VWAP returns, the volatility of closing price returns tends to understate the beta risk estimation result for portfolios. By consequence, the research suggests that by using VWAP along with the closing price, estimation of financial risk and asset pricing can be performed with considerably less noise (Ting, 2006).

The literature presented above has verified the existence of reference-day risk on the S\&P500 as well as for the JSE Top 40 index, even after applying various adjustments to the estimation method for beta. Analogous to the South African studies, the question of whether reference-day risk can be observed on the JSE ALSI beckons. Moreover, where estimation methodologies indicate an improvement in the robustness of beta estimates, none of the estimated betas were empirically tested to verify whether beta estimates adhered to the tenets of the 
CAPM. The empirical evidence presented in the literature also evidences the critical need for a methodology which produces a stable estimate for beta which is independent of the reference day.

This research contributes to this body of literature and proposes a methodology for estimating beta which is intuitively simple, yet differs markedly from the empirical methods tested previously. Additionally, this research aims to redeem the underlying tenet of the CAPM, in attempting to explain a positive risk-reward relationship between the systematic risk parameter, beta, and expected returns.

\section{The sample}

This research uses the daily closing level of the JSE ALSI and closing prices of each of the qualifying shares making up the index between 31 December 1992 and 30 June 2017, sourced from Datastream and Ward and Muller's (2015) style engine. The choice of the JSE ALSI as the selected sample, ensured that $99 \%$ of South Africa's market capitalisation was accounted for in this research (JSE, 2017). Given the longitudinal time-based nature of the research, the qualification of companies to the population applied to any firm which at any stage between 1985 and 2016 had sufficient market capitalisation, irrespective of their eventual state. This eliminated the potential for survivorship bias.

Consistent with the studies by Gonzalez et al (2014), Baker et al (2016), and Carter, Muller and Ward (2017), all share prices included adjustments for any unbundling, mergers, share splits and dividend pay-outs. For all comparisons of standard betas against the estimated VWAP betas, all qualifying companies on the JSE ALSI during the period 3 January 2012 until 31 January 2017 with at least five years historical share price data were considered. This resulted in a total of 136 shares under observation. 


\section{Methodology and results}

\section{Investigating the existence of reference-day risk}

Every month within the sample period has approximately 20 trading days, as the trading of listed shares on the JSE typically excludes weekends and South African public holidays. The daily closing share prices and JSE ALSI levels can therefore be organised per 20 trading days, with the first trading day corresponding to the first working day of the initial month, in a five-year time series. Monthly log returns for each of the qualifying shares on the JSE ALSI and the index are thereafter calculated for the period 3 January 2012 to 31 January 2017. This resulted in a series of monthly returns for each of the 20 trading days, estimated for each of the 136 sampled shares and the JSE ALSI.

Using these data, 20 different estimates of beta were generated for each share, by approximating the slope of the regression between an individual share's returns and the JSE ALSI returns (or equivalently, by using Equation 2). The purpose for estimating share betas for each of the 20 trading days was to understand whether share betas exhibited variation when estimated using the conventional method, but by using 20 different starting points in estimating beta for a given share, within the same sample period.

Consistent with the findings of Gonzalez et al (2014) and Baker et al (2016), first inspection of the conventional beta estimates reveals that there is indeed an effect on beta when the reference day is varied. Using the range of beta across the 20 trading days, reference-day risk is most pronounced in Lonmin (LON), Kumba Iron Ore (KIO) and Royal Bafokeng Platinum (RBP). Similarly, there are also companies for which share betas are relatively constant across the 20 trading days, which includes Remgro (REM), Rebosis Property Fund (REB) and British American Tobacco (BTI).

Alarmingly, 15 out of the 136 sampled companies also exhibited conventional betas which could be classified as either positive or negative by an appropriate choice of the reference day. An equally noteworthy observation is that nine out of ten companies with the highest ranges in betas across the 20 trading days were resources companies. This finding potentially indicates a systemic characteristic in the way resources shares exhibit reference-day risk, or is merely a function of the JSE ALSI being more heavily weighted toward resources shares as compared to other stock exchanges.

Table 1: Smallest and largest ranges in standard betas

Smallest ranges

\begin{tabular}{lccccccc}
\cline { 7 - 8 } & REM & REB & BTI & & LON & KIO & \multicolumn{1}{l}{ RBP } \\
\hline Max & 1.173 & 0.618 & 0.589 & & 5.148 & 3.010 & 1.965 \\
Min & 0.896 & 0.323 & 0.288 & & 2.259 & 0.488 & 0.474 \\
Range & 0.277 & 0.295 & 0.301 & & 2.890 & 2.521 & 1.491 \\
Mean & 1.049 & 0.482 & 0.463 & & 3.486 & 1.776 & 0.985 \\
Variance & 0.005 & 0.006 & 0.010 & & 0.571 & 0.545 & 0.209 \\
Median & 1.052 & 0.471 & 0.492 & & 3.458 & 1.776 & 0.779 \\
\hline
\end{tabular}


As suggested by Acker and Duck (2007), Gonzalez et al (2014) and Baker et al (2016), the differences in betas have profound implications in all its applications. One could consider beta-style portfolio construction as such a case.

Consider the investor with a risk-averse profile intending to construct a portfolio of mainly defensive shares. Such a portfolio will have the characteristics of being weakly correlated with the market and having a positive portfolio beta, which is low in value and less than 1. If such an investor were to estimate the betas for KIO or RBP on a trading day which yields a relatively low beta, such a portfolio may have a positive beta ranging between 0.488 (if the portfolio is constructed entirely of $\mathrm{KIO}$ shares) or 0.474 (if the portfolio is constructed entirely of RBP shares) - varying according to the weightings of each of the shares held. Applying beta to the CAPM, one could therefore expect returns from such a portfolio which have a low correlation with the market. This implies that the investor would realise smaller positive returns when the market moves up, but be shielded from all downward movements in the market.

However, the investor has inadvertently constructed a portfolio with a worst-case scenario beta ranging between 1.965 and 3.010, being the largest betas for RBP and KIO, respectively. Such a portfolio would exhibit extreme fluctuations in value when the market moves in either direction. The systematic risk of the investor's portfolio has been severely misestimated due to reference-day risk. Baker et al (2016) further indicate that an in such a case, an investor may be leisurely to react to a sharp decline in the market, believing his portfolio is weakly correlated with the market.

Equivalent results would be reached for an investor looking to create a portfolio of shares which are negatively correlated with the market. Such an investor may achieve this by including shares with a negative beta in a portfolio. However, the choice of reference day may once again, severely undermine the investors ability to construct such a portfolio, as some shares yield estimates of beta which can be classified as either positive or negative based on the choice of the reference day (E.g. Brimstone Investment Corporation (BRN)). In similar fashion, managing reference-day risk is made even more complex when one is looking to construct a diversified portfolio of shares or when used in the construction of market-neutral hedge funds. In such cases, variations in beta may yield unintended and potentially severe consequences for the performance of the fund.

To statistically determine the degree to which varying the reference day leads to variations in estimated betas, an ANOVA was performed for the 20 trading days. Even though the preliminary analysis of estimated betas across the 20 trading days suggests that there is indeed a trading day effect when estimating share betas, at both the $5 \%$ and $10 \%$ level of significance, the ANOVA reveals insufficient evidence to verify a definitive trading day effect of beta, when the reference day is varied ( $p$-value $=0.501)$. The result is indeed surprising when one considers the large variation in estimated betas exhibited by some shares, suggesting that perhaps the covariance between shares within individual trading days may cancel each other out. Not surprisingly however, a similar result was also reported by Baker et al (2016), who could not statistically verify a trading day effect on beta across their 20 estimates of share betas for the JSE Top 40. Different results are however reached when comparing the minimum and maximum estimated betas for each share.

Each share has a trading day corresponding to its highest beta estimate and equivalently, a trading day which yields its lowest beta estimate. Using a (two-tailed) $t$-test to compare the means of these beta values across firms indicated that the highest and lowest beta values are significantly different at the $5 \%$ level $(p<0.0001)$. This result indicates that for some part, reference-day risk does indeed create additional uncertainty when estimating share betas. Baker et al (2016) also tested this hypothesis and noted a significant difference in minimum and maximum betas for 22 out of the 40 sampled shares. The variation in beta estimates across reference days implies that any application of share betas (and consequently, the CAPM) may severely undermine one's understanding of risk. It may further lead to inefficient share selection when creating portfolios and ultimately, increase the likelihood of sub-optimal investment decisions, especially when valuing companies or constructing market-neutral hedge funds. 


\section{Developing a point estimate of systematic risk for application when reference-day risk is considered}

The primary aim of this research was to develop a more robust point estimate of beta when reference-day risk is exhibited in estimates of systematic risk. Baker et al (2016) presented the potential for using an average of the betas across the 20 trading days as an estimate of systematic risk for a share, but note the potential introduction of errors due to small sample size. In this subsection, the research investigated the feasibility of using a VWAP method for estimating beta when reference-day risk is considered.

Using the total daily value of shares traded together with the number of shares traded for each share on the JSE ALSI, a daily 60-day ex-ante VWAP was estimated for the sample, as per the computation prescribed by Ting (2006);

$$
V W A P=\frac{\sum_{k=1}^{n} P_{k} V_{k}}{V}=\sum_{k=1}^{n} W_{k} P_{k}
$$

(Equation 3)

Where;

$W_{k}$ is the weight and calculated as $V_{k} / V$,

$P_{k}$ are the $n$ intraday prices at which transactions have occurred during the period,

$V$ is the total share volume traded over the period and is equal to $\sum_{k=1}^{n} V_{k}$,

$V_{k}$ are the subtotals of all shares transacted at the price $P_{k}$ (Ting, 2006).

The equation simply states that the VWAP is a combination of all intraday prices, which for the case of this research, occurred over the past 60 days for a share, inclusive of the reference-day. For the JSE ALSI, the VWAP of the index is weighted on a per share basis, and changes daily with movements in the market capitalisation of firms.

Using the VWAP for each share and the index, log returns were computed over a five-year period. Put simply, we estimate share returns (and index returns) monthly by using the VWAP at each month end (instead of the closing price). VWAP betas were then estimated for each share using the conventional methodology for the period 30 March 2012 to 31 March 2017.

At this point, Gonzalez et al (2014) tested the significance of the differences between standard betas against Blume (1971) betas and betas approximated using a student's $t$-distribution method. The authors focused on comparing mean share beta variances for each of the three methods, across 20 trading days. Baker et al (2016) employed a different approach and compared adjusted regression coefficients using the Blume (1971), Dimson (1979) and Vasicek (1973) Bayesian adjustment methods.

This research employed a unique approach in estimating beta which was simple and intuitive, yet distinctly different from the previous investigations on reference-day risk. The conventional method for estimating beta was maintained, however monthly closing prices were substituted with the 60-day ex-ante VWAP. This was done prior to regressing individual share returns against the market returns. Reiterating Ting $(2005 ; 2006)$, the VWAP is statistically more efficient than the closing price in reflecting value as it is closer to the unobservable equilibrium price, resulting in a smaller realised variance, which is the essence of the beta measure.

To understand whether the VWAP beta is a more robust estimate of systematic risk when reference-day risk is considered, VWAP betas were compared to standard betas. To achieve this, both the standard betas and VWAP betas were estimated using the same sample period and tested for statistical difference. This test is critical to the investigation as estimates of VWAP betas employ the same computational methodology as the standard 
beta. Even though the estimation methods are different, the two measures must produce statistically independent estimates of systematic risk. A positive result is therefore fundamental in proposing VWAP as an alternate estimation method in the context of reference-day risk. A paired t-test at the $5 \%$ level of significance confirms that the two measures are statistically different.

Application of the VWAP beta and understanding whether VWAP betas perform better under conditions of reference-day risk

The first step in this analysis was to understand how estimates of VWAP betas for the 136 sampled shares were distributed, and whether tighter estimates of share betas were possible using the conventional method. Furthermore, the research also aimed to understand if there exists a trading day within the 20-day range for which betas tend to be more robust. A visual and statistical comparison was conducted, in analogous fashion to Baker et al (2016), when attempting to understand whether systematic risk estimates using the bootstrapped beta method were more robust for shares on the JSE Top 40 index.

VWAP betas for the sample shares were plotted on a histogram to understand the sample characteristics of estimated share betas, particularly focusing on the skewness and kurtosis of the distribution. Similarly, beta estimates for the sample shares were thereafter plotted using the median beta for each share from the 20 trading days. This process was then repeated for all share betas estimated using the $20^{\text {th }}$ trading day (as this day yielded the tightest distribution in estimated betas). For control, standard betas were also estimated for the sample and equivalently plotted. The resultant distributions were then visually compared. Figure 1 (below) plots each of the distributions for estimates of share betas for the same sample. A positive result would be a normally distributed set of betas, tightly distributed around the mean value, 1 (which represents the market beta).

Figure 1: Distribution of estimated betas

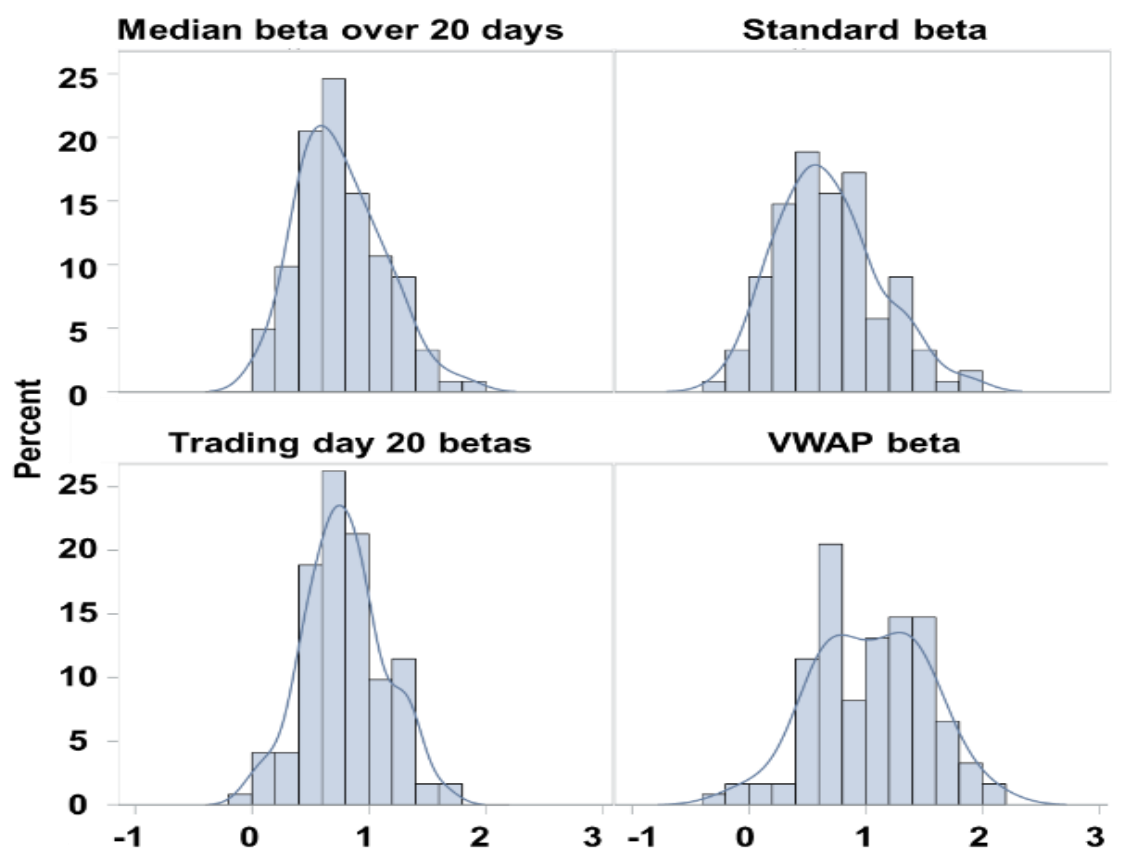

To test the various distributions statistically, the Kolmogorov-Smirnov, Cramer-Von Mises and Anderson-Darling tests were performed to understand whether the estimated betas were normally distributed. Furthermore, the variance for each of the distributions was analysed to determine which method yielded the tightest share beta estimates around the mean, congruent to the method employed by Gonzalez et al (2014). Thereafter, a Levene's 
test was conducted to statistically verify that the variances were distinctly different across the alternate measures, consequently verifying the visual result.

As evident in Figure 1, betas estimated using the $20^{\text {th }}$ trading day of the month produced the most robust estimates in share betas for the sample period, even more so than the standard betas which use month end share values. Counter-intuitively, the VWAP method produces a less robust set of share betas for the sample. This was also verified statistically as described above.

Following this result, there is still the potential for the VWAP betas to be applied in finance. As per the method conducted by Baker et al (2016), beta estimates using the VWAP method were also tested on a per share basis. This is achieved by comparing the distribution of standard beta across the 20 trading days against estimates of beta using the VWAP method. To ensure a robust test of the VWAP beta method on a share by share basis, additional VWAP betas were generated from a non-overlapping, out-of-sample period using a further 10 years historical data (1 January 2002 to 31 January 2012). As conducted prior, differences were tested both visually and statistically.

The results indicate that for most shares, the distributions of VWAP betas are not statistically different from the standard betas. However, upon closer inspection, some positive results were observed when analysing the shares with the largest distributions in standard betas. For the top three shares with the largest ranges in standard beta across the 20 trading days (LON, KIO and RBP), KIO and RBP had statistically different distributions ( $p=0.004$ and $p=0.002$, respectively) for which the VWAP estimates were significantly tighter around the mean. This is illustrated in Figure 2. For LON, this was not the case. Further inspection into LON's share price history does however reveal that the share did experience significant volatility during the sample period, potentially influencing estimates of share betas for the company.

Figure 2: Distribution of estimated betas for Kumba Iron Ore and Royal Bafokeng Platinum

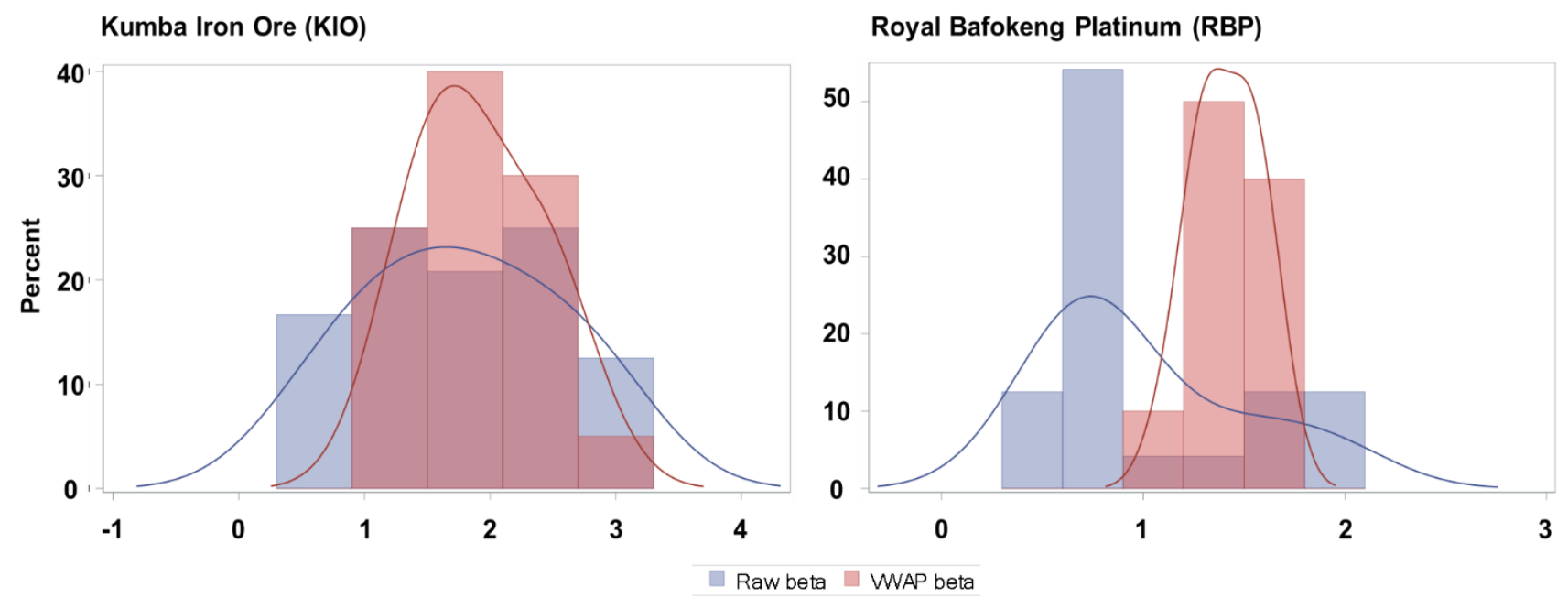

As expected for the three shares with the smallest range in estimated betas (REM, REB and BTI), there was little evidence of a VWAP method improving the range of estimates.

This result suggests that, for cases of extreme reference-day variation in estimated betas, the VWAP beta estimation method does indicate some evidence of a more robust estimate of systematic risk. The VWAP 
method for estimating beta could consequently be applied alongside other measures of systematic risk. This is especially the case when estimating betas for shares which exhibit a large degree of reference-day variation. Furthermore, future research could contemplate the appropriateness of the VWAP beta, when estimating systematic risk for resources shares, as these were evidenced to have the highest effect when the reference day was varied.

\section{Understanding whether the VWAP betas adhere to the tenets of the CAPM}

To consolidate the findings between the standard beta and VWAP beta, the VWAP beta must adhere to the underlying tenet of the CAPM, which states that higher betas would lead to higher returns, and vice versa. This was investigated by means of a graphical time series style analysis, congruent to the methodology employed by Ward and Muller (2012) and Muller and Ward (2013).

To conduct this, each share in the sample was ranked according to the magnitude of the 60-day ex-ante VWAP betas and placed into virtual portfolios in the form of quintiles. Portfolio 1 contained the shares with the highest VWAP betas whereas Portfolio 5 contains the shares with the lowest VWAP betas. The performance of each of the portfolios was thereafter simulated using the Ward and Muller (2015) style engine, which contained share returns data over the period 1985 to 2017 . The simulation was then run over the sample period.

To ensure a robust analysis, each of the VWAP betas for shares within the ALSI are recalculated and ranked every three months. This was done in a similar fashion to that of Carter et al (2017) and ensured that the correct shares were placed in the appropriate portfolios according to their updated betas. The portfolios are rebalanced quarterly on this basis.

Consistent with Ward and Muller (2012), the graphical time series analysis also plots a price relative line to indicate the relationship between quintile 1 (which had shares with the highest VWAP betas) and quintile 5 (which had shares with the lowest VWAP betas). The graphical time series analysis also plots a price relative line between quintile 1 and the market. This acts as a control variable would in the investigation, and is used to test whether high VWAP beta shares lead to abnormal returns (i.e. returns more than what the market could provide), as specified by the CAPM. The performance of the portfolios as well as the relationship between each portfolio and the market was then visually analysed.

Figure 3: Ranked portfolios in terms of VWAP betas

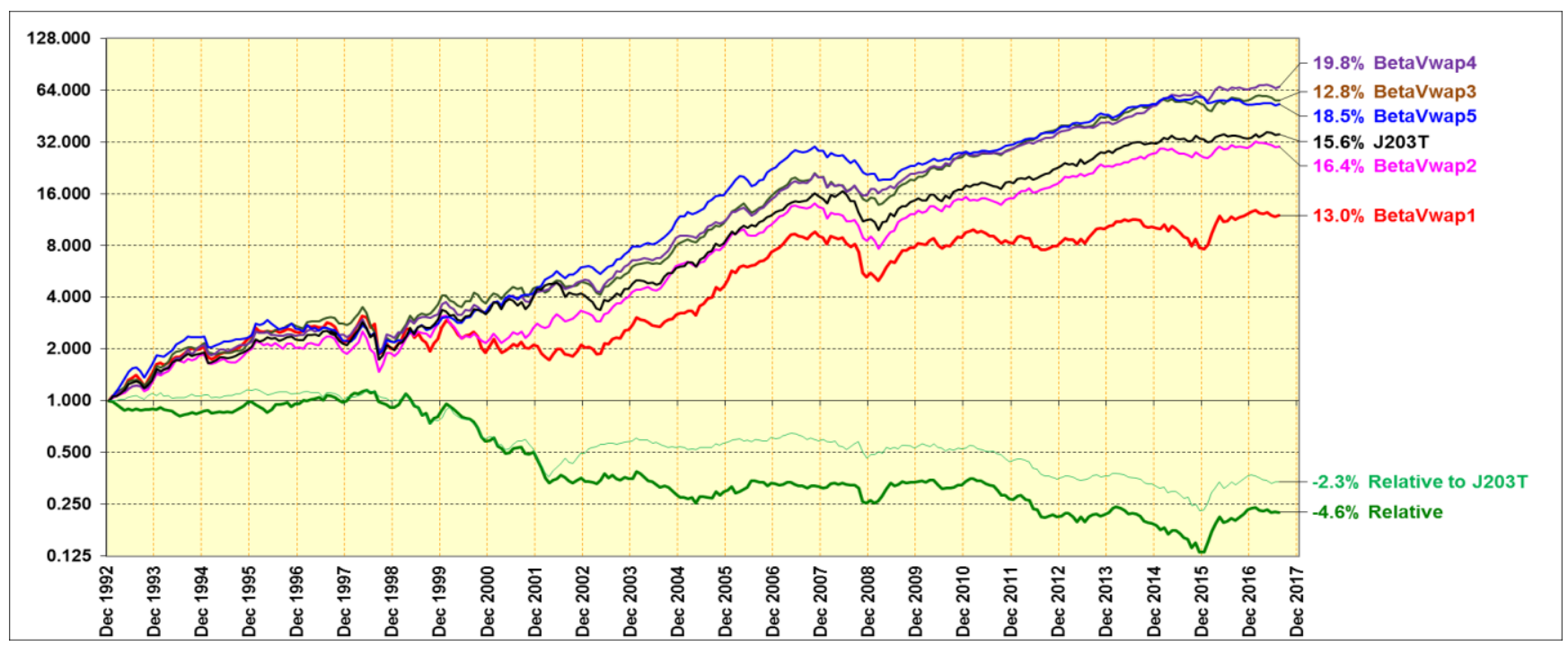


The analysis indicates that the quintiles ranked from highest to lowest, according to the simulated annualised returns, were quintile 4 , quintile 3 , quintile 5 , quintile 2 and lastly, quintile 1 . The price relative further indicates that since December 1999, the quintile with the lowest VWAP betas (quintile 5) consistently outperformed quintile 1, which contained the highest ranked VWAP betas. The data suggest that, even though the VWAP betas are statistically different from the standard betas, the VWAP betas do not adhere to the tenet of the CAPM that a higher level of risk (as estimated by beta) will lead to higher returns, and vice versa. Counter intuitively, the analysis has suggested that shares with a lower VWAP beta tend to outperform shares with high VWAP betas. This is consistent with the findings of Ward and Muller (2012), who found that estimates of standard betas also invert the CAPM.

\section{Conclusion}

This paper set out to resurrect the systematic risk parameter, beta, by contributing a volume-weighted-averageprice (VWAP) point estimate for beta when reference-day risk is considered. This investigation evidenced a significant degree of reference-day risk when estimating share betas on the JSE ALSI, congruous to the findings of Acker and Duck (2007), Dimitrov and Govindaraj (2007), Gonzalez et al (2014) and Baker et al (2016). As a result, this creates additional uncertainty for investors and practitioners applying beta in portfolio construction, risk management, business valuation and all applications of the CAPM. Moreover, this finding underpins the need for a more accurate estimate of systematic risk, when reference-day risk is observed.

VWAP betas were also found to be statistically different from standard betas. Despite the indication from Ting (2005; 2006), there is however limited evidence to suggest that a VWAP method improves estimates of systematic risk for shares. Even so, the investigation recommends that for cases of extreme reference-day variations, the VWAP beta may be applied alongside other measures to improve financial decision making.

Lastly, this research contributes further insight into the risk-relationship trade-off postulated by the CAPM. A graphical time-series style analysis revealed that estimates of beta employing a VWAP methodology still inverts the expected returns. In addition to the empirical findings documented by other researchers, this investigation suggests that the CAPM appears to imperfectly specify the risk-reward trade-off (van Rensburg \& Robertson, 2003; Montier, 2009; Strugnell et al, 2011; Ward \& Muller, 2012). Further research is however required to verify this.

For further research, the investigation evidenced a significant degree of reference day risk when estimating share betas for resources shares. Appreciating that the composition of the JSE ALSI is weighted heavily towards resources shares, further research may potentially uncover additional characteristics in the way resources shares exhibit reference-day risk.

To end, this research also evidenced that for shares on the JSE ALSI, VWAP returns produced more robust covariance matrices for shares. Financial practitioners may find this result useful for many financial applications.

\section{References}

Acker, D., \& Duck, N. W. (2007). Reference-day risk and the use of monthly returns data. Journal of Accounting, Auditing \& Finance, 22(4), 527-557.

Baker, C., Rajaratnam, K., \& Flint, E. J. (2016). Beta estimates of shares on the JSE Top 40 in the context of reference-day risk. Environment Systems and Decisions, 36(2), 126-141.

Blume, M. E. (1971). On the assessment of risk. The Journal of Finance, 26(1), 1-10. 
Brooks, R. D., Faff, R.W., Gangemi, M.A.M., \& Lee, J.H.H. (1997). A further examination of the effect of diversification on the stability of portfolio betas. Applied Financial Economics, Vol. 7, 9-14.

Cademartori, D., Romo, C., Campos, R., \& Galea, M. (2003). Robust estimation of systematic risk using the $t$ distribution in the Chilean stock markets. Applied Economics Letters, 10(7), 447- 453.

Carter, B., Muller, C., \& Ward, M. (2017). The applicability of Black's variation of the capital asset pricing model (CAPM) in the South African context (Master's thesis). Illovo, South Africa: Gordon Institute of Business Science.

Chen, B., \& Reeves, J. J. (2012). Dynamic asset beta measurement. Applied Financial Economics, 22(19), 1655-1664, DOI: 10.1080/09603107.2012.674203.

Collins, D. W., Ledolter, J., \& Rayburn, J. (1987). Some further evidence on the stochastic properties of systematic risk. Journal of Business, 425-448.

Dimitrov, V., \& Govindaraj, S. (2007). Reference-day risk: observations and extensions. Journal of Accounting, Auditing \& Finance, 22(4), 559-572.

Dimson, E. (1979). Risk measurement when shares are subject to infrequent trading. Journal of Financial Economics, 7(2), 197- 226.

Fabozzi, F. J., \& Francis, J. C. (1978). Beta as a random coefficient. Journal of Financial and Quantitative Analysis, 13, 101-15.

Faff, R., \& Brooks, R. (1996). Further evidence on the relationship between beta stability and the length of the estimation period (No. 96-10). Retrieved from: https://ideas.repec.org/p/fth/melrfi/96-10.html

Fama, E. F., \& French, K. R. (1992). The cross section of expected stock returns. Journal of Finance, 47(2), 427- 465.

Fama, E. F., \& French, K. R. (2004). The capital asset pricing model: theory and evidence. Journal of Economic Perspectives, 18(3), 25-46. http://dx.doi.org/10.1257/0895330042162430.

Gonzalez, M., Rodriguez, A., \& Stein, R. (2014). Adjusted betas under reference-day risk. The Engineering Economist, 59(1), 79-88.

Heymans, A., \& Brewer, W. P. (2015). The influence of volatility spill- overs and market beta on portfolio construction. South African Journal of Economic and Management Sciences, 18(3), 277- 290.

JSE. (2017). What is the JSE All Share Index (ALSI)? Retrieved from: https://www.jse.co.za/grow-mywealth/what-is-the-jse-all-share- index.

Lintner, J. (1965). The Valuation of Risk Assets and the Selection of Risky Investments in Stock Portfolios and Capital Budgets. The Review of Economics and Statistics, 47(1), 13-17. http://doi.org/10.2307/1924119.

Markowitz, H. M. (1952). Portfolio Selection. The Journal of Finance, 7(1), 77. http://doi.org/10.2307/2975974.

Markowitz, H.M. (1956). The optimization of a quadratic function subject to linear constraints. Naval Research Logistics Quarterly, 3(1- 2):111-133.

Markowitz, H.M. (1959). Portfolio selection: Efficient diversification of investments. New York: Wiley.

Montier, J. (2009). Behavioural Investing: a practitioner's guide to applying behavioural finance. John Wiley \& Sons. 
Mossin, J. (1966). Equilibrium in a Capital Asset Market. Econometrica, 34(4), 768-783.

http://doi.org/10.2307/1910098.

Muller, C., \& Ward, M. (2013). Style-based effects on the Johannesburg Stock Exchange: A graphical timeseries approach. Investment Analysts Journal, 77, 1-16.

Novak, J. (2015). Systematic risk changes, negative realized excess returns and time-varying CAPM beta. Journal of Economics and Finance, 65, 2015 (2), 167-190.

Sharpe, W. F. (1964). Capital Asset Prices: A Theory of Market Equilibrium under Conditions of Risk. The Journal of Finance, 19(3), 425-442. http://doi.org/10.2307/2977928.

Strugnell, D., Gilbert, E., \& Kruger, R. (2011). Beta, size and value effects on the JSE, 19942007. Investment Analysts Journal, 40(74), 1-17.

Ting, C. (2005). Which daily price is more efficient? (Vol. 1). Working paper, Singapore Management University. Retrieved from: https://www.researchgate.net/profile/Christopher_Ting/publ ication/260399763_Which_Daily_Price_is_More_Efficient/li nks/53d5c5d70cf228d363ea103a.pdf.

Ting, C. (2006). Which daily price is less noisy?. Financial Management, 35(3), 81-95.

Treynor, J. (1961). Market value, time, and risk. Unpublished manuscript dated 08/08/1961, No. 95-209.

Van Rensburg, P., \& Robertson, M. (2003). Size, price-to-earnings and beta on the JSE Securities Exchange. Investment Analysts Journal, 32(58), 7-16.

Vasicek, O. A. (1973). A Note on Using Cross-sectional Information in Bayesian Estimation of Security Betas. The Journal of Finance, 28(5), 1233-1239.

Ward, M., \& Muller, C. (2012). Empirical Testing of the CAPM on the JSE. Investment Analysts Journal, 76, $1-12$.

Ward, M., \& Muller, C. (2015). The Risk-Free Rate and the CAPM on the JSE. Unpublished, 1-7. 